\title{
Downregulation of microRNA-196a enhances the sensitivity of non-small cell lung cancer cells to cisplatin treatment
}

\author{
QIAN LI $^{1 *}$, ZAILIANG YANG $^{1 *}$, MINGYAN CHEN ${ }^{1}$ and YING LIU ${ }^{2}$ \\ ${ }^{1}$ Department of Respiratory Medicine, The Sixth People's Hospital of Chongqing; \\ ${ }^{2}$ Department of Respiratory Medicine, Southwest Hospital, Chongqing, P.R. China
}

Received March 7, 2015; Accepted February 12, 2016

DOI: $10.3892 /$ ijmm.2016.2513

\begin{abstract}
MicroRNAs (miRNAs or miRs) are a class of small, non-coding RNA molecules that play an important role in the pathogenesis of human diseases through the regulation of gene expression. Although miRNA-196a has been implicated in the progression of human lung cancer, its role in enhancing the sensitivity of non-small cell lung cancer (NSCLC) cells to cisplatin has not yet been confirmed. The aim of this study was to evaluate the effects of miRNA-196a on the sensitivity of NSCLC cells to cisplatin in vitro and in vivo. RT-qPCR was used to detect miRNA-196a expression. Synthesized locked nucleic acid (LNA)-anti-miRNA-196a oligonucleotide was transiently transfected into the SPC-A-1 and A549 lung cancer cells to examine the effects of miRNA-196a on the growth of and colony formation inthe cisplatin-treated cells. The effects of miRNA-196a on the sensitivity of SPC-A-1 cells to cisplatin in vivo were determined using $\mathrm{BALB} / \mathrm{c}$ nude mice. The expression of miRNA-196a was significantly higher in both the lung cancer tissues and cell lines. The LNA-based knockdown of miRNA-196a significantly inhibited SPC-A-1 and A549 cell growth and induced apoptosis. Moreover, the downregulation of miRNA-196a sensitized the SPC-A-1 and A549 NSCLC cells to cisplatin in vitro and in vivo, by inducing apoptosis. The findings of this study demonstrate that the administration of cisplatin in combination with miRNA-196a-targeted therapy may be a potential therapeutic strategy for the treatment of NSCLC.
\end{abstract}

\section{Introduction}

Lung cancer is the leading cause of cancer-related mortality worldwide $(1,2)$, and non-small cell lung cancer (NSCLC) accounts for the majority of all lung cancer-related deaths (3).

Correspondence to: Dr Qian Li, Department of Respiratory Medicine, The Sixth People's Hospital of Chongqing, 301 Nancheng Avenue, Nanan, Chongqing 400060, P.R. China

E-mail:danw_zj@126.com

*Contributed equally

Key words: microRNA-196a, non-small cell lung cancer cells, cisplatin, sensitivity, apoptosis
The prognosis of lung cancer remains unfavorable, with a 5 -year overall survival rate of approximately $11 \%$, despite recent advances in clinical and experimental oncology (4). Thus, detailed research into the development and progression of NSCLC is essential for improving the diagnosis, prevention and treatment of this disease. Cisplatin (DDP) remains the most widely used first-line chemotherapeutic agent for the treatment of NSCLC. However, the continuous and multiple-dose administration of DDP often causes severe side-effects and cancer cells often become resistant; thus, this has limited the use of this drug (5). Therefore, enhancing the sensitivity of cancer cells to DDP (perhaps to lower doses of the drug) remains a challenge for the efficacty of chemotherapy. Recently, an increasing number of studies has demonstrated that small, non-coding RNAs may be involved in the pathogenesis of $\operatorname{NSCLC}(6,7,14)$, thereby providing new insight into the biology of the disease. Previous studies have indicated that the dysregulation of microRNAs (miRNAs or miRs) contributes to the resistance of human cancer cells to DDP $(8,9)$. Novel therapeutic modalities combining miRNAs have the potential to be effective in the treatment of NSCLC in the future.

miRNA-196a (miR-196a) was one of the first miRNAs to be discovered in human cells; it is highly conserved in mammals. Previous studies have indicated that the expression of miR-196a is significantly upregulated in different solid tumors (10-12) and have revealed that miR-196a is involved in the proliferation, detachment, migration and invasion of a number of cancer cells (colorectal cancer, breast cancer, pancreatic cancer, gastric cancer and NSCLC cells) (12-16). Huang et al reported that miR-196a promotes the progression of pancreatic cancer (13); Liu et al found that the downregulation of miR-196a inhibited NSCLC cell proliferation and invasion (14). However, to the best of our knowledge, there are no studies available to date on the association between miR-196a expression and the sensitivity of NSCLC cells to DDP.

In the present study, we found that miR-196a was upregulated in human NSCLC tissues and cell lines; the downregulation of miR-196a enhanced the sensitivity of NSCLC cell lines (SPC-A-1, A549) to DDP through the induction of apoptosis by targeting homeobox A5 (HOXA5). Taken together, these findings suggest that miR-196a is a valid therapeutic target with the potential to be employed as a novel multimodality therapy as part of a strategy for the treatment of patients with NSCLC. 


\section{Materials and methods}

Patients and tissue samples. A total of 23 pairs of matched NSCLC and non-cancerous tissue samples were obtained from patients undergoing surgical procedures at the Sixth People's Hospital of Chongqing (Chongqing, China), and tumor diagnosis was performed by an independent pathologist. None of the patients had received chemotherapy or radiotherapy prior to surgery. The samples were snap-frozen in liquid nitrogen and stored at $-80^{\circ} \mathrm{C}$ until RNA extraction. Written informed consent was obtained from all patients prior to surgery. This study was approved by the Research Ethics Committee of the Sixth People's Hospital of Chongqing, China.

Animals. Female BALB/c nude mice ( $\mathrm{n}=48$, 4-6 weeks of age) were purchased from the Shanghai Laboratory Animal Research Center (Shanghai, China) and maintained under pathogen-free conditions. All experimental procedures involving animals were performed in accordance with the Guide for the Care and Use of Laboratory Animals and were performed according to the Institutional Ethical Guidelines for Animal Experiments.

Cell culture. Four NSCLC adenocarcinoma cell lines (A549, SPC-A-1 , NCI-H1650 and NCI-H1299), a NSCLC squamous carcinoma cell line (SK-MES-1) and a normal human bronchial epithelial cell line (16HBE) were purchased from the Institute of Biochemistry and Cell Biology of the Chinese Academy of Sciences (Shanghai, China). The cells were cultured in RPMI-1640 medium supplemented with $10 \%$ fetal bovine serum (FBS) (both from Gibco, Grand Island, NY, USA), $100 \mathrm{U} / \mathrm{ml}$ penicillin and $100 \mathrm{mg} / \mathrm{ml}$ streptomycin (both from Invitrogen, Carlsbad, CA, USA) in humidified air with $5 \% \mathrm{CO}_{2}$ at $37^{\circ} \mathrm{C}$.

Locked nucleic acid (LNA)-anti-miR-196a oligonucleotidetransfection assay. The SPC-A-1 and A549 cells were maintained in RPMI-1640 medium. For transfection, LNA-antimiR-196a or LNA-control oligonucleotides were delivered at a final concentration of $50 \mathrm{nM}$ [as previously described (17)] using Lipofectamine 2000 reagent (Invitrogen). LNA-antimiR-196a and LNA-control oligonucleotides were purchased from Exiqon A/S (Vedbaek, Denmark). A group of mock cells (untransfected cells) was also used.

Treatment of cells with DDP. The mock SPC-A-1/A549 cells and the SPC-A-1/A549 cells transfected with LNA-anti-miR-196a/ LNA-control oligonucleotide were treated with various concentrations $(0,5,10$ and $20 \mu \mathrm{g} / \mathrm{ml})$ of DDP for $12 \mathrm{~h}$ or $5 \mu \mathrm{g} / \mathrm{ml}$ of DDP for $0,24,48,72$ and $96 \mathrm{~h}$.

Cell viability assay. Cell viability was assessed using the CCK8 assay (Beyotime Institute of Biotechnology, Haimen, China) according to the manufacturer's instructions. Briefly, the treated cells were cultured in triplicate in a 96-well plate. CCK8 reagent was added to each well $2 \mathrm{~h}$ prior to the termination of the experiment, and the absorbance (OD450) was expressed as the viability percentages of the cells compared with the controls. All tests were performed in triplicate and the data are presented as the means $\pm \mathrm{SD}$.
Reverse transcription-quantitative polymerase chain reaction ( $R T-q P C R)$. Total RNA was extracted from the mocktransfected SPC-A-1/A549 or stably-transfected SPC-A-1/ A549 cells $\left(5 \times 10^{6}\right.$ cells $)$ and the tissue samples, using TRIzol reagent (Invitrogen) according to the manufacturer's instructions. To quantify the miRNA levels, RT-qPCR was performed using TaqMan microRNA assays (Applied Biosystems Life Technologies, Foster City, CA, USA). Briefly, $10 \mathrm{ng}$ of total RNA was reverse transcribed using an miRNA-specific looped RT primer for each miRNA and a corresponding TaqMan ${ }^{\circledast}$ microRNA Reverse Transcription kit (Applied Biosystems Life Technologies; prime sequences included in kit). qPCR was performed using the generated cDNA in genespecific TaqMan miRNA Real-Time PCR assay solution on a StepOnePlus Real-Time PCR system (Applied Biosystems Life Technologies). The reaction was performed at $95^{\circ} \mathrm{C}$ for $10 \mathrm{~min}$, followed by 45 cycles at $95^{\circ} \mathrm{C}$ for $15 \mathrm{sec}$, and $60^{\circ} \mathrm{C}$ for $60 \mathrm{sec}$. RNA U6 (RNU6B; Applied Biosystems Life Technologies) was used as an internal control. The relative expression was calculated using the comparative cycle threshold $(\mathrm{Ct})$ method. All qPCR reactions were performed in triplicate and the data are presented as the means $\pm \mathrm{SD}$.

Colony formation assay. Approximately 500 mock-transfected SPC-A-1/A549 or stably-transfected SPC-A-1/A549 cells were placed in a fresh 6-well plate with or without DDP for $12 \mathrm{~h}$ and maintained in RPMI-1640 containing 10\% FBS for another 2 weeks. The colonies were fixed with methanol and stained with $0.1 \%$ crystal violet in $20 \%$ methanol for $15 \mathrm{~min}$. All samples were analyzed in triplicate and the data are presented as the means $\pm \mathrm{SD}$.

Flow cytometric analysis of apoptosis. The mock-transfected SPC-A-1/A549 or stably-transfected SPC-A-1/A549 cells were treated with DDP and harvested. The cells were double-stained with FITC-Annexin V and propidium iodide (PI), and then analyzed using a flow cytometer equipped with CellQuest software (both from BD Biosciences, Franklin Lakes, NJ, USA), as previously described (18). The relative ratio of apoptotic cells was compared with the control from each experiment. All samples were analyzed in triplicate and the data are presented as the means $\pm \mathrm{SD}$.

Caspase-3 activity assay. Briefly, the cells were seeded in a 6-well plate, cultured for $24 \mathrm{~h}$ and then treated with or without DDP for a further $12 \mathrm{~h}$, and harvested. The activity of caspase-3 was measured using a Caspase-3 Colorimetric assay kit (Sigma-Aldrich, St. Louis, MO, USA) according to the manufacturer's instructions. Caspase-3 activity was quantified spectrophotometrically at a wavelength of $405 \mathrm{~nm}$. All samples were analyzed in triplicate and the data are presented as the means \pm SD.

Western blot analysis. Protein extracts $(40 \mu \mathrm{g})$ from the treated cells were separated by $15 \%$ sodium dodecyl sulfate-polyacrylamide gel electrophoresis (SDS-PAGE; Invitrogen) and electrophoretically transferred onto PDVF membranes (GE Healthcare Life Sciences, Pittsburgh, PA, USA). The membranes were blocked with 5\% non-fat dried milk for $2 \mathrm{~h}$, and then incubated for $2 \mathrm{~h}$ with specific primary antibodies 

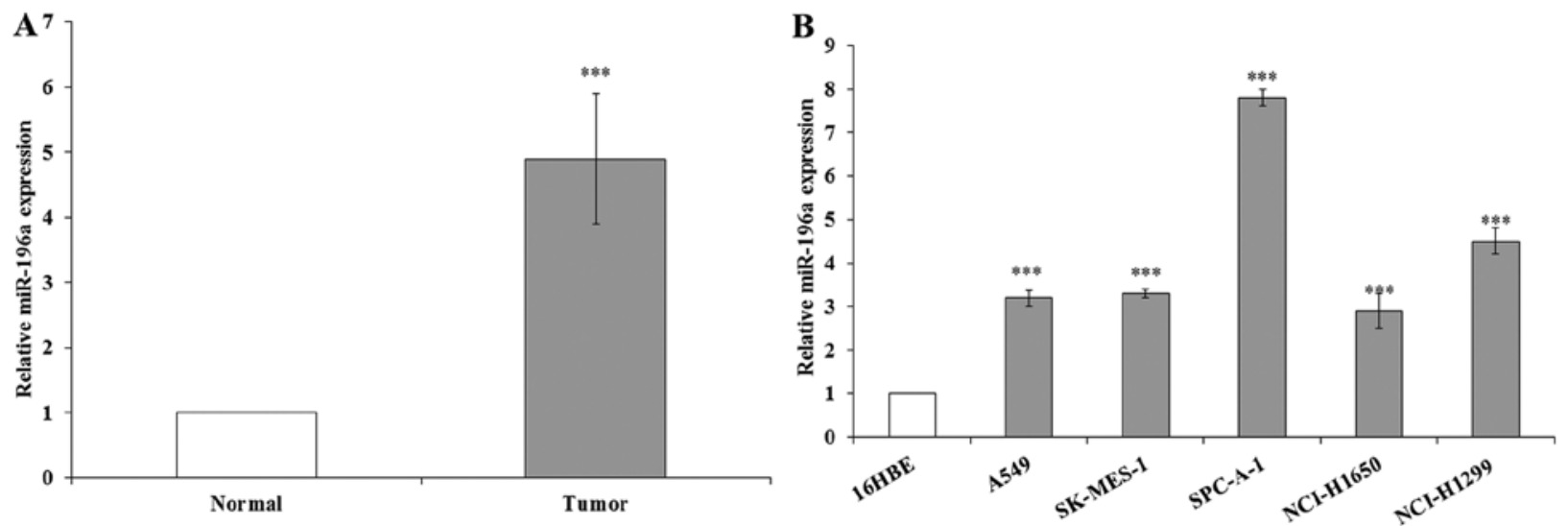

Figure 1. RT-qPCR analysis of miR-196a expression in non-small cell lung cancer (NSCLC) tissues and cell lines. (A) Analysis of miR-196a expression in NSCLC tissues. miR-196a was detected in 23 pairs of NSCLC tissues by RT-qPCR. Data are presented as fold changes in tumor tissues relative to normal tissues. (B) Analysis of miR-196a expression levels in NSCLC cell lines compared with the normal bronchial epithelial cell line (16HBE) by RT-qPCR. All experiments were performed in biological triplicate with 3 technical replicates. ${ }^{* * *} \mathrm{p}<0.001$ compared to normal tissues and cells .

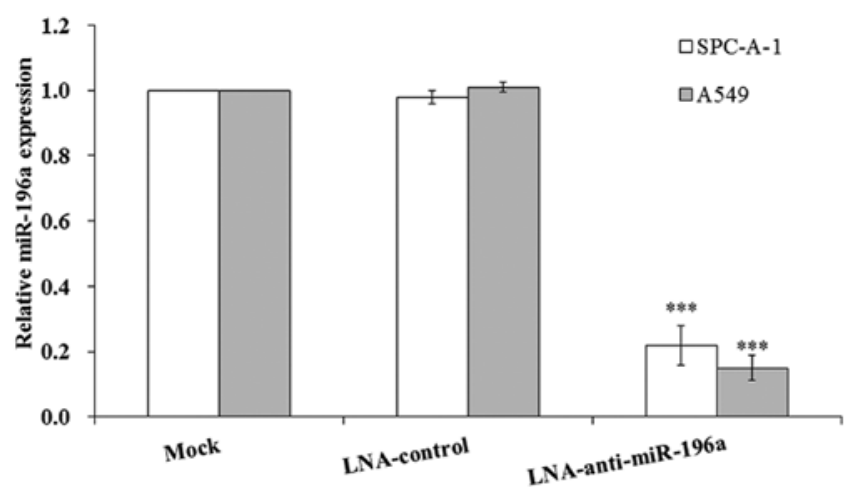

Figure 2. RT-qPCR analysis of miR-196a expression following transfection of the SPC-A-1/A549 cells with locked nucleic acid (LNA)-anti-miR-196a or LNA-control oligonucleotide. All experiments were performed in biological triplicate with 3 technical replicates. ${ }^{* * *} \mathrm{p}<0.001$.

HOXA5 (sc-365784), p53 (sc-126) and $\beta$-actin (sc-376421) (all from Santa Cruz Biotechnology, Santa Cruz, CA, USA). After washing with TBST (10 mM Tris, $\mathrm{pH} 8.0,150 \mathrm{mM} \mathrm{NaCl}$, and $0.1 \%$ Tween-20), the membranes were incubated with horseradish peroxidase-linked antibody (\#7076; Cell Signaling Technology, Inc., Danvers, MA, USA) for $1 \mathrm{~h}$. The membranes were washed and the proteins were visualized using ECL chemiluminescence and exposed to X-ray film. All of the samples were analyzed in triplicate.

In vivo experiments using mice. $\mathrm{BALB} / \mathrm{c}$ nude mice were randomly divided into 6 groups [mock + vehicle (normal saline), mock + DDP, LNA-NC + vehicle, LNA-NC + DDP, LNA-anti-miR196a + vehicle and LNA-anti-miR196a + DDP; 8 mice/group]. The mice were injected with either mock-transfected or stably-transfected SPC-A-1 cells by subcutaneous injection $\left(3 \times 10^{6}\right.$ cells $\left./ 0.2 \mathrm{ml}\right)$. One day after tumor cell implantation, the mice were treated with DDP $[3.0 \mathrm{mg} / \mathrm{kg}$; intraperitoneally (i.p.) every other day (qod)] or the vehicle. Tumor volume was examined for 5 weeks and measured once a week. The volume formed was calculated using the following formula: volume $=0.4 \times \mathrm{D} \mathrm{x} \mathrm{d}^{2}(\mathrm{D}$, longitudinal diameter; $\mathrm{d}$, latitudinal diameter). All mice were sacrificed by exposure to carbon dioxide and the tumors were harvested, and TUNEL staining assay was then performed.

TUNEL staining assay. The tissues were harvested and plated on polylysine-coated slides. The slides were then fixed with $4 \%$ paraformaldehyde for $1 \mathrm{~h}$ at room temperature, and were then rinsed with $0.1 \mathrm{M}$ PBS, and finally permeabilized with $1 \%$ Triton X-100. DNA fragmentation was detected using the TUNEL Apoptosis Detection kit (KeyGen, Nanjing, China), which specifically labeled 3'-hydroxyl termini of DNA strand breaks using fluorescein isothiocyanate (FITC)-conjugated dUTP. The DNA was labeled with FITC DNA-binding dye for $5 \mathrm{~min}$. The FITC fluorescence was measured using a fluorescence microscope. The percentage of apoptotic cells was calculated as the number of apoptotic cells per the number of total cells $\mathrm{x} 100$.

Statistical analysis. In the present study, the data are expressed as the means \pm SD. Statistical analysis was performed using a t-test with SPSS 13.0 software to evaluate the significance of differences between groups. A p-value $<0.05$ was considered to indicate a statistically significant difference.

\section{Results}

miR-196a expression is upregulated in human NSCLC tissues and cell lines. In this study, the miR-196a levels were measured in 23 NSCLC samples and adjacent normal tissues by RT-qPCR, and normalized to U6. The results indicated that miR-196a expression was significantly upregulated in the NSCLC samples compared with the expression levels in the corresponding normal tissue samples (Fig. 1A). We also performed RT-qPCR to examine the expression of miR-196a in the human NSCLC cell lines, including both adenocarcinoma and squamous carcinoma subtypes. The results revealed that miR-196a expression was also significantly upregulated in the human NSCLC cell lines compared with the expression levels in the normal cell line, 16HBE (Fig. 1B). These results indicate that the overexpression of miR-196a may play an important role in the progression and development of NSCLC. 

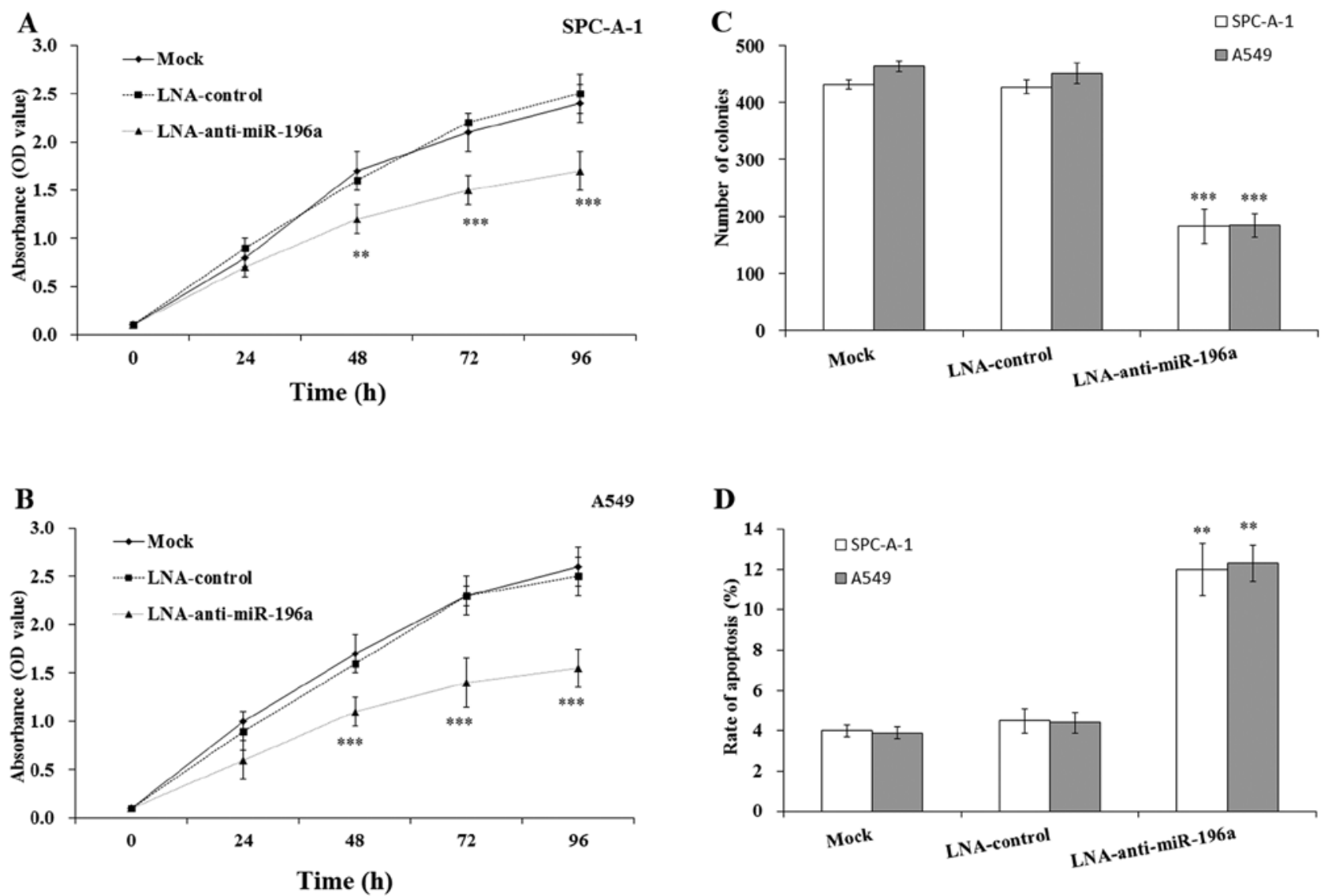

Figure 3. Effects of miR-196a on SPC-A-1/A549 cell proliferation and apoptosis. (A) SPC-A-1 cells and (B) A549 cells were transfected with locked nucleic acid (LNA)-anti-miR-196a or LNA-control oligonucleotide. CCK8 assay was performed to analyze the proliferation of SPC-A-1 cells. (C) Colony formation assays were performed to analyze the proliferation of SPC-A-1/A549 cells. The number colonies was counted. (D) Flow cytometric analysis of the apoptosis of SPC-A-1/ A549 cells transfected with LNA-anti-miR-196a or LNA-control oligonucleotide. ${ }^{* *} \mathrm{p}<0.01$ and ${ }^{* * *} \mathrm{p}<0.001$.

Manipulation of miR-196a levels in NSCLC cells by performing LNA-anti-miR-196a oligonucleotide transfection assay. To selectively downregulate miR-196a in the NSCLC cell lines, SPC-A-1/A549, the LNA-anti-miR-196a oligonucleotide transfection assay was used in this study. The SPC-A-1/A549 cells were transfected with LNA-anti-miR-196a or LNA-control oligonucleotide; $48 \mathrm{~h}$ after transfection, the cells were harvested and RT-qPCR was performed. The results revealed that the expression of miR-196a was significantly downregulated by approximately 4-5-fold following transfection with LNA-antimiR-196a oligonucleotide compared with that in the mock-transfected cells (Fig. 2); therefore, transfection with LNA-anti-miR-196a oligonucleotide was used to manipulate the miR-196a level to investigate the biological effects of miR-196a in the subsequent experiments.

Effect of miR-196a on NSCLC cell proliferation and apoptosis. To examine the biological role of miR-196a in NSCLC cells, we examined the effects of downregulating miR-196a on cell proliferation and apoptosis by CCK8 assay, colony formation assay and FACS analysis. As shown in Fig. 3A and B, the SPC-A-1 and A549 cells transfected with LNA-anti-miR-196a oligonucleotide exhibited a significant decrease in cell viability compared with the mock- or LNA-control-transfected cells, particularly after $96 \mathrm{~h}(\mathrm{p}<0.001)$. Similarly, the results of colony formation assay revealed that clonogenic survival was decreased following the downregulation of miR-196a in the SPC-A-1 and A549 cells compared with the the mock- or LNA-control-transfected cells $(\mathrm{p}<0.001$; Fig. $3 \mathrm{C}$ ). To determine whether apoptosis was a contributing factor to cell growth inhibition, we performed flow cytometric analysis of the SPC-A-1 and A549 cells following transfection with LNA-anti-miR-196a oligonucleotide. The results revealed that the apoptotic rate was significantly increased in the SPC-A-1/A549 cells transfected with LNA-anti-miR-196a oligonucleotide compared with that in the mock- or LNA-controltransfected cells $(\mathrm{p}<0.01)$ (Fig. 3D). Taken together, these results indicate that the inhibition of miR-196a may inhibit the growth and induce the apoptosis of SPC-A-1 and A549 cells.

Downregulation of miR-196a enhances the sensitivity of NSCLC cells to DDP in vitro. The dysregulation of miRNA expression has been reported to be associated with chemoresistance in human cancers (19). However, whether miR-196a expression affects the sensitivity of NSCLC cells to DDP is not yet fully understood. In the present study, we hypothesized that there was an association between the dysregulation of miR-196a and the sensitivity of NSCLC cells to DDP. To examine this hypothesis, the mock-transfected SPC-A-1/A549 cells and the SPC-A-1/A549 cells transfected with LNA-anti-miR-196a/LNA-control oligonucleotide were treated with various concentrations $(0,5,10$ and $20 \mu \mathrm{g} / \mathrm{ml})$ of DDP for $12 \mathrm{~h}$ or $5 \mu \mathrm{g} / \mathrm{ml}$ of DDP for $0,24,48,72$ and $96 \mathrm{~h}$. 

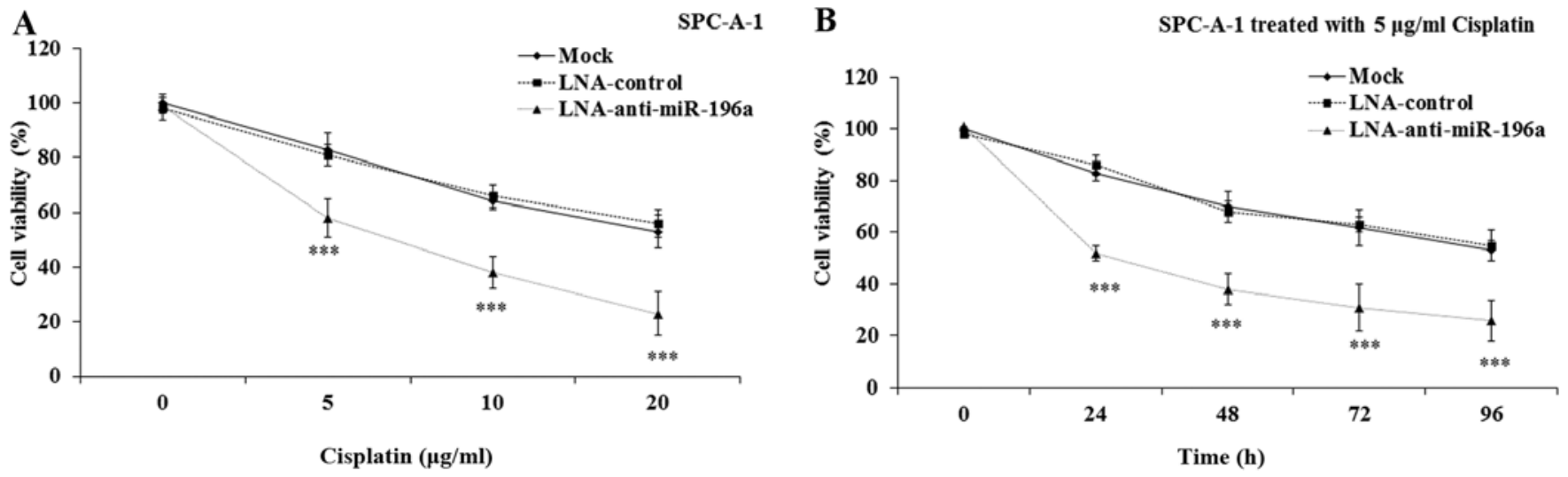

A549
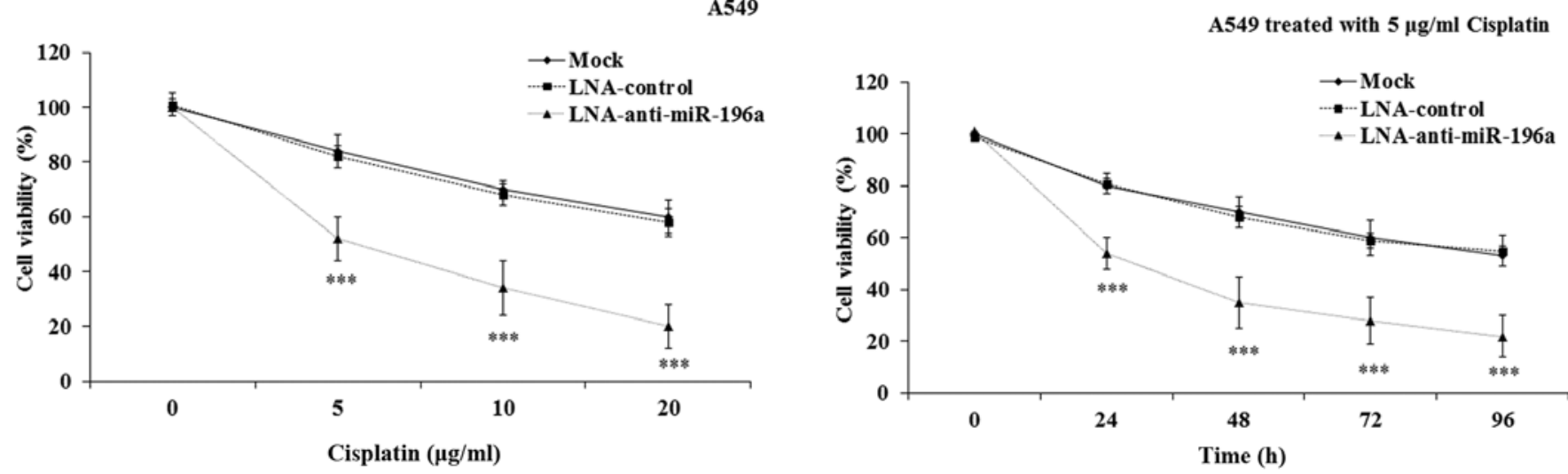

Figure 4. Effect of miR-196a downregulation on the sensitivity of SPC-A-1/A549 cells to cisplatin (DDP) in vitro. (A) Effects of treating the cells (SPC-A-1/A549 cells) with various concentrations of DDP for $12 \mathrm{~h}$ were assessed by CCK8 assay. (B) Effects of treating the cells (SPC-A-1/A549 cells) with $5 \mu \mathrm{g} / \mathrm{ml}$ DDP for various periods of time were evaluated by CCK8 assay. All experiments were performed in triplicate. ${ }^{* * *} \mathrm{p}<0.001$.
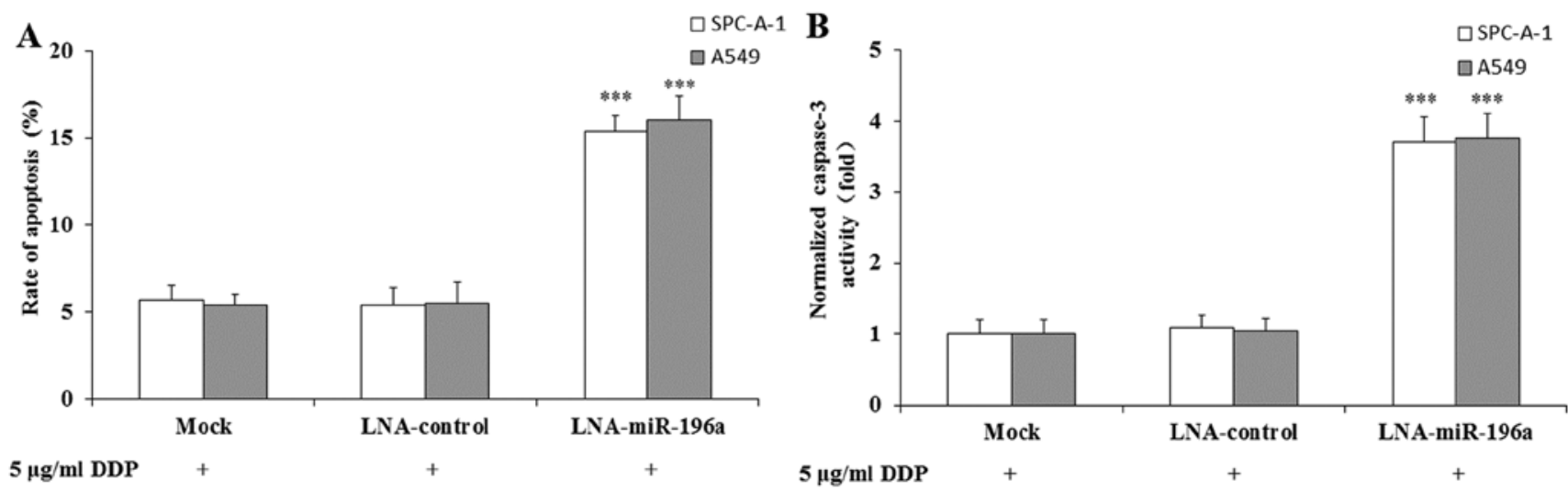

Figure 5. Effect of the combination of miR-196a downregulation with DDP $(5 \mu \mathrm{g} / \mathrm{ml})$ treatment on the apoptosis of SPC-A-1/A549 cells. (A) Flow cytometric analysis of the apoptosis of SPC-A-1/A549 cells. (B) Analysis of relative caspase-3 activity in SPC-A-1/A549 cells transfected with locked nucleic acid (LNA)anti-miR-196a or LNA-control oligonucleotide. All experiments were performed in triplicate. ${ }^{* * *} \mathrm{p}<0.001$.

The CCK8 assay was performed to determine cell viability. The results indicated that the downregulation of miR-196a led to a significant decrease in the viability of the SPC-A-1/A549 cells treated with DDP, in a dose- and time-dependent manner compared with that in the LNA-control and the mock-transfected cells $(p<0.001$; Fig. 4). These data clearly demonstrate that the downregulation of miR-196a may effectively enhance the sensitivity of NSCLC cells to DDP.
Downregulation of miR-196a enhances the DDP-induced apoptosis of NSCLC cells. To determine whether apoptosis was a contributing factor to the enhanced sensitivity of SPC-A-1/ A549 cells to DDP, we performed flow cytometric analysis. As shown in Fig. 5A, the apoptotic rate was significantly increased in the SPC-A-1/A549 cells transfected with LNA-anti-miR-196a oligonucleotide and treated with $5 \mu \mathrm{g} / \mathrm{ml}$ DDP compared with that in the mock-transfected cells treated with $5 \mu \mathrm{g} / \mathrm{ml}$ DDP. 


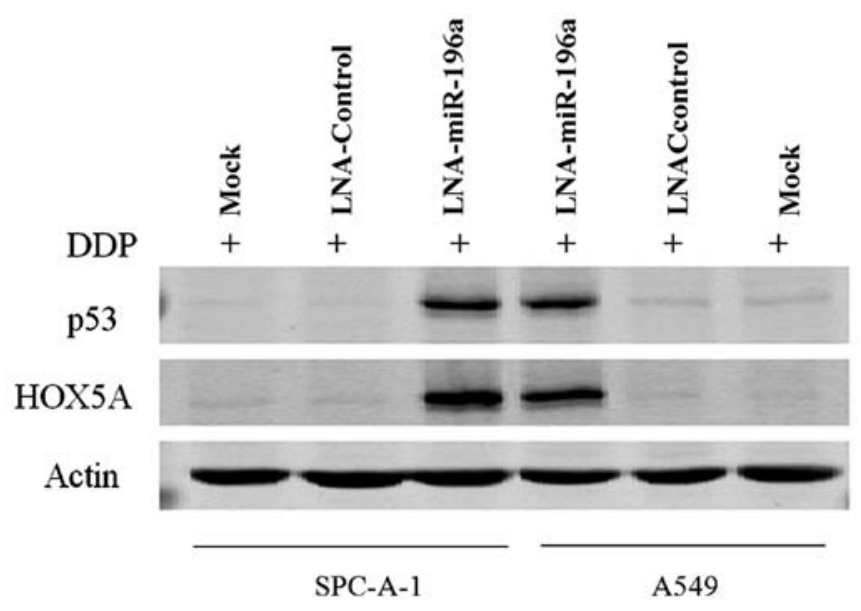

Figure 6. Effects of miR-196a downregulation on the cisplatin (DDP)-induced apoptotic signaling pathway. Western blot analysis of HOXA5 and p53 in SPC-A-1/A549 cells. All experiments were performed in triplicate.

The apoptotic rate of the SPC-A-1/A549 cells transfected with LNA-control oligonucleotide and treated with DDP did not differ significantly compared with that of the mock-transfected cells treated with DDP. We then examined caspase- 3 activity using a colorimetric assay. The results revealed that caspase-3 activity in the SPC-A-1/A549 cells transfected with LNA-antimiR-196a oligonucleotide and treated with DDP significantly increased compared with that in the mock- or LNA-controltransfected cells treated with DDP (Fig. 5B). Therefore, the downregulation of miR-196a may increase the sensitivity of SPC-A-1/A549 cells to DDP by enhancing DDP-induced apoptosis.

Downregulation of miR-196a enhances HOXA5 expression in NSCLC cells. A previous study indicated that miR-196a promotes NSCLC cell proliferation and invasion by targeting HOXA5 (14). Raman et al reported that the overexpression of HOXA5 induced cell apoptosis and the overexpression of p53 concomitantly (20). Thus, to determine whether the apoptosis induced by the downregulation of miR-196a was affected by HOXA5, we performed western blot analysis to determine the HOXA5 and p53 levels in the cisplatin-treated NSCLC cells. The results revealed that the downregulation of miR-196a enhanced the expression of HOXA5 and p53 in the SPC-A-1/A549 cells treated with $5 \mu \mathrm{g} / \mathrm{ml}$ DDP (Fig. 6). RT-qPCR was then performed to determine the mRNA levels of HOXA5 and p53, and the results of RT-qPCR were similar to those of western blot analysis (data not shown). Therefore, the downregulation of miR-196a induced the expression of HOXA5 and p53 at the protein and mRNA level. Taken together, these findings suggest that the downregulation of miR-196a enhances the sensitivity of NSCLC cells to DDP through apoptotic signaling by targeting HOXA5. We aim to elucidate the precise mechanism of this interaction with apoptotic signaling in future studies.

Downregulation of miR-196a enhyances the sensitivity of SPC-A-1 cells to DDP in vivo. To examine the effects of downregulating miR-196a on the sensitivity of SPC-A-1 cells to DDP in vivo, nude mice were subcutaneously injected with SPC-A-1 cells to form tumors. This was followed by the administration of either DDP or the vehicle. The results revealed that the tumors formed following transfection with LNA-anti-miR-196a oligonucleotide grew significantly slower than those from the mice injected with the mock- or LNA-control-transfected SPC-A-1 cells (Fig. 7A). Following treatment with DDP, the inhibition of tumor growth in the mice injected with the SPC-A-1 cells transfected with LNA-anti-miR-196a oligonucleotide was much greater than that in the mice injected with mock- or LNA-control-transfected SPC-A-1 cells $(\mathrm{p}<0.05)$. The results of TUNEL assay revealed that the apoptotic rate in the tumors of mice injected with the SPC-A-1 cells transfected with LNA-anti-miR-196a oligonucleotide was significantly higher than that of the tumors from the mice injected with the mock- or LNA-control-transfected SPC-A-1 cells following treatment with DDP $(\mathrm{p}<0.001$; Fig. 7B). These data clearly indicate that the downregulation of miR-196a may effectively enhance the sensitivity of SPC-A-1 cells to DDP by inducing apoptosis in vivo.
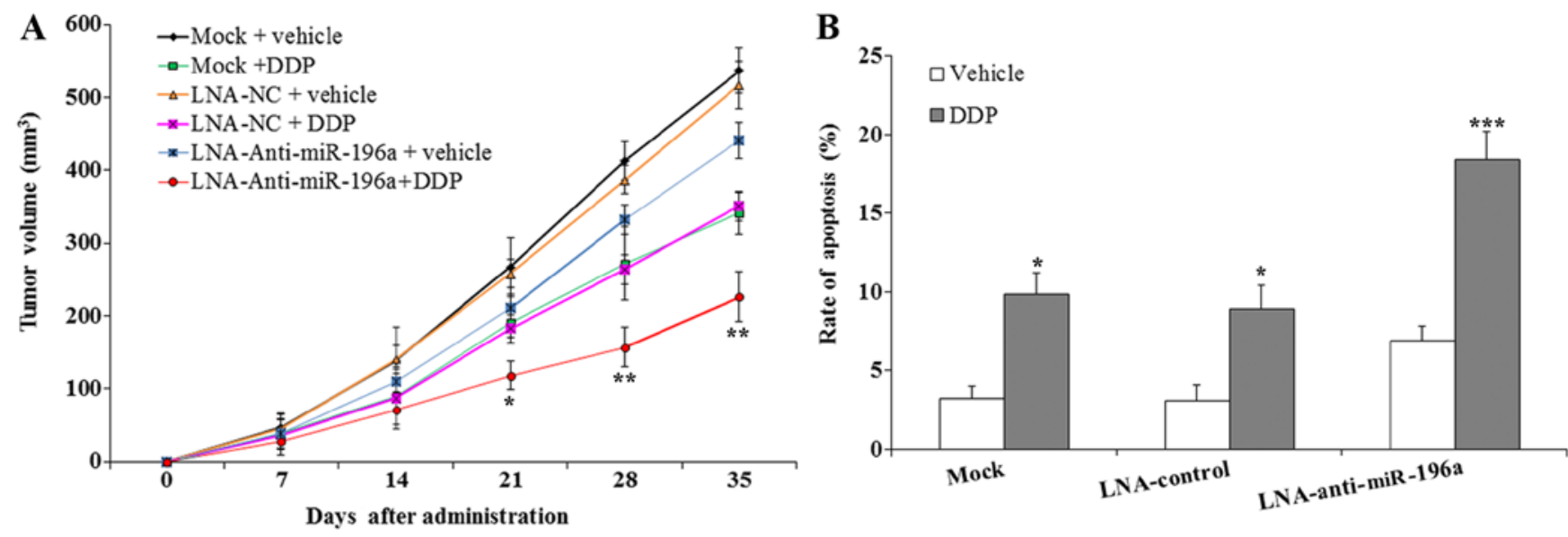

Figure 7. Effects of miR-196a downregulation on the sensitivity of SPC-A-1 cells to cisplatin (DDP) in vivo. (A) Growth of tumors in mice injected with SPC-A-1 cells transfected with locked nucleic acid (LNA)-anti-miR-196a or LNA-control oligonucleotide with or without DDP treatment. (B) TUNEL staining assay of apoptosis in tumor tissues on day 35 after the inoculation of SPC-A-1 cells with or without DDP treatment ( $\mathrm{n}=8$ mice/group). ${ }^{*} \mathrm{p}<0.05,{ }^{* * *} \mathrm{p}<0.01$ and ${ }^{* * * *} \mathrm{p}<0.001$. 


\section{Discussion}

It is well known that miRNAs are a series of small (19-24 nt in length), non-coding RNAs which are involved in posttranscriptional gene regulation or degradation $(21,22)$. In a wide range of plant and animal cells, miRNAs have been shown to play an important role in various processes, including cell proliferation, differentiation and metabolism $(23,24)$. They bind to target mRNAs at the 3'-untranslated region (UTR) and/ or 5'-UTR of target mRNA, to block translation or contribute to target mRNA degradation (25). There is increasing evidence to suggest that the deregulation of miRNAs frequently occurs in tumor tissues, and that they target genes involved in cancer cell proliferation, differentiation, apoptosis, metastasis and resistance (26-29). It has also been demonstrated that miRNAs play an important role in modulating sensitivity and resistance to anticancer drugs in substantial ways (19).

Recently, the function of miR-196a in the pathogenesis of tumors has been widely investigated; a number of studies have suggested that miR-196a exhibits an oncogenic function in cancer $(30-33,40)$. Higher levels of miR-196a have been found in pancreatic cancer, leukemia and esophageal adenocarcinoma, and have been shown to be negatively associated with survival (34-38). In esophageal cancer, miR-196a overexpression has been shown to promote cell proliferation and to suppress apoptosis by directly regulating annexin A1 (39). In colorectal cancer, high levels of miR-196a have been shown to promote cancer cell detachment, migration, invasion and chemosensitivity, and to promote the development of lung metastases in mice by activating the Akt signaling pathway $(11,40)$. However, the mechanisms responsible for the chemosensitivity mediated by miR196a have not yet been clearly defined and evidence of an association between miR-196a and chemoresistance remains limited. In addition, the potential involvement of miR-196a in the induction of drug resistance, particularly in resistance to DDP, remains to be determined. Inspired by the above-mentioned observations, in this study, we investigated the biological role of miR-196a in mediating resistance to DDP in NSCLC cells.

In this study, we demonstrated that miR-196a expression was upregulated in NSCLC tissue samples and NSCLC cell lines. In addition, we attempted to explore the role of miR-196a in NSCLC; the results revealed that the targeted knockdown of miR-196a expression in NSCLC cells led to the significant inhibition of cell proliferation and colony formation by inducing caspase-3-dependent apoptosis. Furthermore, we found that the downregulation of miR-196a significantly enhanced the sensitivity of NSCLC cells to DDP in vitro and in vivo, by targeting HOXA5. However, further studies exploring the role of miR-196a in the regulation of NSCLC cell growth and metastasis are warranted in the future.

miR-196a plays critical roles in the pathogenesis of cancer by targeting several genes, including high mobility group AT-hook 2 (HMGA2), annexin A1 and HOX; however, miR-196a is not only expressed in cancer cells, but also in normal cells (40-43), and the functions and targets of miR-196a have not yet been fully analyzed. The downregulation of miR-196a may induce abnormal gene expression in normal cells. As a potential concern, the miRNA antagonist used may also non-specifically bind to other RNAs, and one miRNA may have multiple target genes due to the sequence homology of the binding sites, which may result in unwanted side-effects. It has been reported that single-nucleotide polymorphism (SNP) in miRNA-196a is associated with severe toxicity in lung cancer patients, particularly in individuals treated with cisplatin or gemcitabine (44). In spite of the multiple potential side-effects, miR-196a gene therapy may prove to be a novel multimodality treatment for NSCLC with continued research.

Taken together, the findings of the present suggest that the downregulation of miR-196a enhances the sensitivity of NSCLC cells to DDP both in vitro and in vivo. Thus, the appropriate application of DDP in combination with the regulation of miR-196a expression may prove to be a potential therapeutic strategy for the treatment of NSCLC in the future.

\section{References}

1. World Cancer Report 2014. World Health Organization. Chapter 1.1, 2014.

2. Mehlen P and Puisieux A: Metastasis: a question of life or death. Nat Rev Cancer 6: 449-458, 2006.

3. Jemal A, Siegel R, Xu J and Ward E: Cancer statistics, 2010. CA Cancer J Clin 60: 277-300, 2010.

4. Verdecchia A, Francisci S, Brenner H, Gatta G, Micheli A, Mangone L and Kunkler I; EUROCARE-4 Working Group: Recent cancer survival in Europe: a $2000-02$ period analysis of EUROCARE-4 data. Lancet Oncol 8: 784-796, 2007.

5. Kostova I: Platinum complexes as anticancer agents. Recent Pat Anticancer Drug Discov 1: 1-22, 2006.

6. Liu Y, Li M, Zhang G and Pang Z: MicroRNA-10b overexpression promotes non-small cell lung cancer cell proliferation and invasion. Eur J Med Res 18: 41, 2013.

7. Ma Q, Jiang Q, Pu Q, Zhang X, Yang W, Wang Y, Ye S, Wu S, Zhong G, Ren J, et al: MicroRNA-143 inhibits migration and invasion of human non-small-cell lung cancer and its relative mechanism. Int J Biol Sci 9: 680-692, 2013.

8. Yu ZW, Zhong LP, Ji T, Zhang P, Chen WT and Zhang CP: MicroRNAs contribute to the chemoresistance of cisplatin in tongue squamous cell carcinoma lines. Oral Oncol 46: 317-322, 2010.

9. Sorrentino A, Liu C-G, Addario A, Peschle C, Scambia G and Ferlini C: Role of microRNAs in drug-resistant ovarian cancer cells. Gynecol Oncol 111: 478-486, 2008.

10. Hui AB, Shi W, Boutros PC, Miller N, Pintilie M, Fyles T, McCready D, Wong D, Gerster K, Waldron L, et al: Robust global micro-RNA profiling with formalin-fixed paraffin-embedded breast cancer tissues. Lab Invest 89: 597-606, 2009.

11. Wang YX, Zhang XY, Zhang BF, Yang CQ, Chen XM and Gao HJ: Initial study of microRNA expression profiles of colonic cancer without lymph node metastasis. J Dig Dis 11: 50-54, 2010.

12. Sun M, Liu XH, Li JH, Yang JS, Zhang EB, Yin DD, Liu ZL, Zhou J, Ding Y, Li SQ, et al: MiR-196a is upregulated in gastric cancer and promotes cell proliferation by downregulating p27(kip1). Mol Cancer Ther 11: 842-852, 2012.

13. Huang F, Tang J, Zhuang X, Zhuang Y, Cheng W, Chen W, Yao H and Zhang S: MiR-196a promotes pancreatic cancer progression by targeting nuclear factor kappa-B-inhibitor alpha. PLoS One 9: e87897, 2014.

14. Liu XH, Lu KH, Wang KM, Sun M, Zhang EB, Yang JS, Yin DD, Liu ZL, Zhou J, Liu ZJ, et al: MicroRNA-196a promotes non-small cell lung cancer cell proliferation and invasion through targeting HOXA5. BMC Cancer 12: 348, 2012

15. Du W, Ma XL, Zhao C, Liu T, Du YL, Kong WQ, Wei BL, Yu JY, Li YY, Huang JW, et al: Associations of single nucleotide polymorphisms in miR-146a, miR-196a, miR-149 and miR-499 with colorectal cancer susceptibility. Asian Pac J Cancer Prev 15: 1047-1055, 2014.

16. Hoffman AE, Zheng T, Yi C, Leaderer D, Weidhaas J, Slack F, Zhang Y, Paranjape T and Zhu Y: microRNA miR-196a-2 and breast cancer: a genetic and epigenetic association study and functional analysis. Cancer Res 69: 5970-5977, 2009.

17. Zhu H, Zhu X, Cheng G, Zhou M and Lou W: Downregulation of microRNA-21 enhances radiosensitivity in nasopharyngeal carcinoma. Exp Ther Med 9: 2185-2189, 2015.

18. Zhang SZ, Pan FY, Xu JF, Yuan J, Guo SY, Dai G, Xue B, Shen WG, Wen CJ, Zhao DH and Li CJ: Knockdown of c-Met by adenovirus-delivered small interfering RNA inhibits hepatocellular carcinoma growth in vitro and in vivo. Mol Cancer Ther 4: 1577-1584, 2005. 
19. Garofalo $M$ and Croce CM: MicroRNAs as therapeutic targets in chemoresistance. Drug Resist Updat 16: 47-59, 2013.

20. Raman V, Martensen SA, Reisman D, Evron E, Odenwald WF, Jaffee E, Marks J and Sukumar S: Compromised HOXA5 function can limit p53 expression in human breast tumours. Nature 405: 974-978, 2000.

21. Ambros V: The functions of animal microRNAs. Nature 431: 350-355, 2004.

22. Bartel DP: MicroRNAs: Genomics, biogenesis, mechanism, and function. Cell 116: 281-297, 2004

23. He H, Jazdzewski K, Li W, Liyanarachchi S, Nagy R, Volinia S, Calin GA, Liu CG, Franssila K, Suster S, et al: The role of microRNA genes in papillary thyroid carcinoma. Proc Natl Acad Sci USA 102: 19075-19080, 2005.

24. Voorhoeve PM, le Sage C, Schrier M, Gillis AJ, Stoop H, Nagel R, Liu YP, van Duijse J, Drost J, Griekspoor A, et al: A genetic screen implicates miRNA-372 and miRNA-373 as oncogenes in testicular germ cell tumors. Cell 124: 1169-1181, 2006.

25. Wu L, Fan J and Belasco JG: MicroRNAs direct rapid deadenylation of mRNA. Proc Natl Acad Sci USA 103: 4034-4039, 2006.

26. Amaral JD, Xavier JM, Steer CJ and Rodrigues CM: Targeting the p53 pathway of apoptosis. Curr Pharm Des 16: 2493-2503, 2010.

27. Dykxhoorn DM: MicroRNAs and metastasis: little RNAs go a long way. Cancer Res 70: 6401-6406, 2010.

28. Zimmerman AL and Wu S: MicroRNAs, cancer and cancer stem cells. Cancer Lett 300: 10-19, 2011.

29. Hummel R, Hussey DJ and Haier J: MicroRNAs: predictors and modifiers of chemo- and radiotherapy in different tumour types. Eur J Cancer 46: 298-311, 2010.

30. Chen C, Zhang Y, Zhang L, Weakley SM and Yao Q: MicroRNA-196: critical roles and clinical applications in development and cancer. J Cell Mol Med 15: 14-23, 2011.

31. Suh YE, Raulf N, Gäken J, Lawler K, Urbano TG, Bullenkamp J, Gobeil S, Huot J, Odell E and Tavassoli M: MicroRNA-196a promotes an oncogenic effect in head and neck cancer cells by suppressing annexin A1 and enhancing radioresistance. Int J Cancer 137: 1021-1034, 2015.

32. Yang G, Han D, Chen X, Zhang D, Wang L, Shi C, Zhang W, Li C, Chen X, Liu H, et al: MiR-196a exerts its oncogenic effect in glioblastoma multiforme by inhibition of I $\mathrm{B} \alpha$ both in vitro and in vivo. Neuro Oncol 16: 652-661, 2014.

33. Zhang J, Zheng F, Yu G, Yin Y and Lu Q: miR-196a targets netrin 4 and regulates cell proliferation and migration of cervical cancer cells. Biochem Biophys Res Commun 440: 582-588, 2013.

34. Bloomston M, Frankel WL, Petrocca F, Volinia S, Alder H, Hagan JP, Liu CG, Bhatt D, Taccioli C and Croce CM: MicroRNA expression patterns to differentiate pancreatic adenocarcinoma from normal pancreas and chronic pancreatitis. JAMA 297 1901-1908, 2007.
35. Szafranska AE, Doleshal M, Edmunds HS, Gordon S, Luttges J, Munding JB, Barth RJ Jr, Gutmann EJ, Suriawinata AA, Marc Pipas J, et al: Analysis of microRNAs in pancreatic fine-needle aspirates can classify benign and malignant tissues. Clin Chem 54: 1716-1724, 2008.

36. Wang J, Chen J, Chang P, LeBlanc A, Li D, Abbruzzesse JL, Frazier ML, Killary AM and Sen S: MicroRNAs in plasma of pancreatic ductal adenocarcinoma patients as novel blood-based biomarkers of disease. Cancer Prev Res (Phila) 2: 807-813, 2009.

37. Wang Y, Li Z, He C, Wang D, Yuan X, Chen J and Jin J: MicroRNAs expression signatures are associated with lineage and survival in acute leukemias. Blood Cells Mol Dis 44: 191-197, 2010.

38. Niinuma T, Suzuki H, Nojima M, Nosho K, Yamamoto H, Takamaru H, Yamamoto E, Maruyama R, Nobuoka T, Miyazaki Y, et al: Upregulation of miR-196a and HOTAIR drive malignant character in gastrointestinal stromal tumors. Cancer Res 72: 1126-1136, 2012.

39. Luthra R, Singh RR, Luthra MG, Li YX, Hannah C, Romans AM, Barkoh BA, Chen SS, Ensor J, Maru DM, et al: MicroRNA-196a targets annexin A1: A microRNA-mediated mechanism of annexin A1 downregulation in cancers. Oncogene 27: 6667-6678, 2008.

40. Schimanski CC, Frerichs K, Rahman F, Berger M, Lang H, Galle PR, Moehler M and Gockel I: High miR-196a levels promote the oncogenic phenotype of colorectal cancer cells. World J Gastroenterol 15: 2089-2096, 2009.

41. Hu Z, Chen J, Tian T, Zhou X, Gu H, Xu L, Zeng Y, Miao R, Jin G, Ma H, et al: Genetic variants of miRNA sequences and non-small cell lung cancer survival. J Clin Invest 118: 2600-2608, 2008.

42. Hu Z, Liang J, Wang Z, Tian T, Zhou X, Chen J, Miao R, Wang Y, Wang $X$ and Shen H: Common genetic variants in premicroRNAs were associated with increased risk of breast cancer in Chinese women. Hum Mutat 30: 79-84, 2009.

43. Yekta S, Shih IH and Bartel DP: MicroRNA-directed cleavage of HOXB8 mRNA. Science 304: 594-596, 2004.

44. Zhan X, Wu W, Han B, Gao G, Qiao R, Lv J, Zhang S, Zhang W, Fan W, Chen H, et al: Hsa-miR-196a2 functional SNP is associated with severe toxicity after platinum-based chemotherapy of advanced nonsmall cell lung cancer patients in a Chinese population. J Clin Lab Anal 26: 441-446, 2012. 